\title{
Pharmacodynamic Comparison of the Carbapenems Against E. coli and Klebsiella spp. Containing Extended spectrum $\beta$-lactamases
}

\author{
${ }^{1}$ Kathryn J. Eagye, ${ }^{1}$ Joseph L. Kuti and ${ }^{1,2}$ David P. Nicolau \\ ${ }^{1}$ Center for Anti-Infective Research and Development, Hartford Hospital, Hartford, Connecticut, USA \\ ${ }^{2}$ Division of Infectious Diseases, Hartford Hospital, Hartford, Connecticut, USA
}

\begin{abstract}
Carbapenems are the treatment of choice for extended-spectrum $\beta$-lactamases (ESBLs). Ertapenem is a new member of the carbapenem class, and is often grouped with imipenem and meropenem as a recommended treatment for ESBLs. However, new in vitro data question ertapenem's inclusion as a first line treatment for these bacteria, suggesting that ertapenem has a lower likelihood of obtaining appropriate pharmacodynamic exposure than other carbapenems. This study-part of the OPTAMA (Optimizing Pharmacodynamic Target Attainment using a Microbiologic Antibiogram) Program-used Monte Carlo simulations to compare cumulative fraction of response (CFR) for carbapenems against ESBL producing isolates of Escherichia coli and Klebsiella species collected in North America. Pharmacokinetic parameters were derived from the literature; MIC distributions were obtained from the 2004 MYSTIC database and two urban teaching hospitals. Pharmacodynamic endpoints evaluated included free drug time above the MIC $\left(f \mathrm{~T}_{>\text {MIC }}\right)$. Ertapenem dosed at 1 gram every 24 hours showed a CFR of $98.1 \%$ at $40 \% \mathrm{f}_{>\mathrm{MIC}}$ against all ESBLs included in 5,000 trials, versus $100.0 \%$ for imipenem and meropenem dosed at 1 gram every 8 hours $(\mathrm{p}<0.001)$. Ertapenem also exhibited a lower CFR against E. coli and Klebsiella when modeled against those species individually (96.9\% and $98.7 \%$, respectively) than the other carbapenems $(100.00 \%$ for all regimens) $(p<0.001)$. Ertapenem demonstrated a probability of target attainment at $40 \% f \mathrm{~T}_{>\text {MIC }}$ of $77 \%$ against bacteria with MICs of $=2 \mu \mathrm{g} / \mathrm{ml}$. All three carbapenems showed high probabilities of achieving bactericidal target attainment against ESBL producing organisms, though ertapenem's lower CFR suggests that it might be a second choice agent in institutions with high rates of resistance among ESBLs.
\end{abstract}

Key words: ESBL, pharmacodynamics, carbapenems, Monte Carlo simulation

\section{INTRODUCTION}

Although $\beta$-lactam antibiotics are among the most widely used antibiotics in hospitals today, emerging resistance in Gram-negative bacteria pressures the continuation of their success. The most common resistance mechanism in Gram-negative bacteria includes production of $\beta$-lactamases that hydrolyze the antibiotic ring, thus deactivating antimicrobial activity $^{[1]}$ In the late 1970s, broad-spectrum cephalosporins were developed to overcome $\beta$ lactamase resistance. Soon after, the earlier narrow spectrum $\beta$-lactamases mutated and Enterobacteriaceae producing extended spectrum $\beta$-lactamases (ESBLs) were reported ${ }^{[2]}$

ESBLs are able to hydrolyze $\beta$-lactams that possess an oxyamino side chain such as ceftazidime, ceftriaxone, and cefotaxime, as well as aztreonam. ${ }^{[3]}$ Additionally, they are often resistant to numerous other antibiotic classes, such as fluoroquinolones and aminoglycosides. Currently, the treatment of choice for
ESBL producing organisms is the carbapenem class (imipenem and meropenem), since they are more stable to hydrolysis by ESBLs. Clinical studies also report a significantly higher success rate for carbapenems over other antimicrobials. ${ }^{[4]}$

Ertapenem is a new once-daily member of the carbapenem class. It exhibits microbiologic activity similar to meropenem, with the exception of poor activity against non-fermenting bacteria such as Pseudomonas aeruginosa and Acinetobacter species. ${ }^{[5]}$ Since it is less expensive than the other carbapenems, is conveniently dosed, and because it may avoid unnecessary selective pressure against $P$. aeruginosa, many hospitals find ertapenem to be an attractive alternative to the older carbapenems for the treatment of ESBLs. This decision is currently based only upon ertapenem's inclusion in the carbapenem class, as data examining ertapenem's clinical success against ESBL producing organisms have not yet been reported. However, recent in vitro data suggest that ertapenem may not be as stable to hydrolysis by ESBLs as the other carbapenems. ${ }^{[6-8]}$ These data question whether 
ertapenem should be advocated as a first line treatment for ESBL producing bacteria.

In the absence of clinical data evaluating the performance of ertapenem against ESBLs specifically, a proxy method is necessary to determine its utility for the treatment of these pathogens. An analysis of the drug's microbiological success against a population of clinically isolated ESBL producing bacteria is a relevant and assessable measure. ${ }^{[9-11]}$ Since standard susceptibility test results of a given drug against a target bacteria do not always correlate well with clinical outcomes, microbiological success is better evaluated as the ability of the antibiotic to achieve appropriate pharmacodynamic exposure (i.e., bactericidal exposure) against the target organism using a typical dosing regimen.

The methodology used in the Optimizing Pharmacodynamic Target Attainment using a Microbiologic Antibiogram (OPTAMA) Program is well-suited to evaluate the performance of the carbapenems against ESBLs. The OPTAMA Program examines the probability of typical antimicrobial regimens of obtaining appropriate pharmacodynamic exposure against common nosocomial pathogens. Previous OPTAMA studies have been conducted using 2002 isolates of E. coli, Klebsiella species, Acinetobacter baumannii, and P. aeruginosa collected from North America, South America, and Europe. ${ }^{[12-14]}$ These studies observed that the carbapenems (i.e., imipenem and meropenem) demonstrated a high likelihood of achieving bactericidal exposure against these bacteria. However, no ESBL producing organisms were identified among the Enterobacteriaceae during that examination period. Moreover, ertapenem had only recently been approved for use and was not included in the examination.

The current study simulated standard regimens of all three carbapenems against nationally collected Escherichia coli and Klebsiella species that produce ESBLs. The purpose of this effort was to illuminate possible differences among these antibiotics in their ability to achieve bactericidal pharmacodynamic exposure in order to assist in the clinical and formulary decision processes.

\section{METHODS}

Pharmacodynamic model. Pharmacodynamic exposures, as measured by the percent of the dosing interval during which free (i.e., unbound) drug remained above the MIC $\left(f \mathrm{~T}_{>\mathrm{MIC}}\right)$, were modeled against all isolates using a Monte Carlo simulation as described below. A one-compartment intravenous (IV)-infusion equation was used to calculate $f \mathrm{~T}_{>\mathrm{MIC}}$ at steady-state. ${ }^{[15]}$ Dosage regimens were chosen based on the most typical practices in North America, and were modeled as follows: thirty-minute IV-infusion regimens of imipenem 1 gram ( $1 \mathrm{~g})$ every eight hours (q8h), meropenem $1 \mathrm{~g} \mathrm{q} 8 \mathrm{~h}$, and ertapenem $1 \mathrm{~g}$ q24h.

Microbiology. Microbiology data used during the pharmacodynamic analyses were derived from three sources: 1) the Meropenem Yearly Susceptibility Test Information Collection (MYSTIC) database; 2) Hartford Hospital (Hartford, CT); and 3) Rush Presbyterian Medical Center (Chicago, IL). The MYSTIC Program is a multi-center surveillance study that compares the activity of meropenem with that of other antimicrobials-including imipenem and ertapenem (as well as compounds from other classes of antibiotics) - against nosocomial isolates of both Grampositive and Gram-negative organisms collected from around the world. [16] MICs are recorded and the isolates are stored for further evaluation.

The data supporting the present study represent isolates collected consecutively from patients hospitalized in North America during the 2004 edition of the MYSTIC Program. ESBLs identification was performed phenotypically using standard CLSI techniques. ${ }^{[17]}$ Not all institutions participating in the MYSTIC Program isolated the ESBL producing organisms of interest during 2004; as a result, the institutions represented in this analysis include only the 11 MYSTIC sites where ESBLs were present. They are distributed geographically across the United States. In addition, two institutions that are not participants in the MYSTIC Program-Hartford Hospital and Rush Presbyterian Medical Center-were included in this examination in order to increase the sample size of ESBLs. Isolates from these two hospitals were collected between 2002 and 2004. A total of 52 E. coli and 79 Klebsiella species (i.e. Klebsiella pneumoniae and oxytoca) isolated at all 13 sites both produced extended spectrum $\beta$-lactamases and had susceptibility data against carbapenems available for analysis. Multiple isolates of the same species from a single origin (same patient) were excluded. Each participant laboratory performed identification at the species level by colony morphology or simple biochemical tests (spot indole, bile solubility, oxidase, etc.) or Vitek ID cards (bioMerieux, Hazelwood, MO) when required.

The MICs of all antibiotics were determined by either broth microdilution or E-test according to CLSI methodology. ${ }^{[17]}$ The detailed methodology for MIC determination has been published elsewhere. ${ }^{[16]}$ MICs ranged from $\leq 0.008 \mu \mathrm{g} / \mathrm{ml}$ to $16 \mu \mathrm{g} / \mathrm{ml}$ in doubling dilutions for all antibiotics. MIC values less than $0.008 \mu \mathrm{g} / \mathrm{ml}$ were classified as $0.008 \mu \mathrm{g} / \mathrm{ml}$. Reported MICs that fell within intervals (e.g., between 0.032 and 0.064) were rounded up to the next highest tube dilution; since the true value of such an MIC could be anywhere within that interval, but is known to be greater than the lower boundary, assigning the upper boundary of the interval is the more conservative approach. The percentage of isolates at each MIC are 
Table 1: MIC distributions for carbapenems tested against ESBL producing bacteria isolated at 13 hospitals in North America.

\begin{tabular}{|c|c|c|c|c|c|c|c|c|c|c|c|c|c|}
\hline \multirow{2}{*}{$\begin{array}{c}\text { Species (no.) or } \\
\text { antimicrobial agent }\end{array}$} & \multicolumn{13}{|c|}{$\%$ of isolates at MIC $(\mu \mathrm{g} / \mathrm{ml})$ of ${ }^{\mathrm{a}}$ : } \\
\hline & 0.008 & 0.016 & 0.032 & 0.064 & 0.125 & 0.25 & 0.5 & 1 & 2 & 4 & 8 & 16 & $\begin{array}{c}\% \\
\text { Susceptible }^{b}\end{array}$ \\
\hline \multicolumn{14}{|l|}{ E. coli (52) } \\
\hline Imipenem & & & & 3.8 & 32.7 & 46.2 & 13.5 & & 3.8 & & & & 100.0 \\
\hline Meropenem & & 21.2 & 28.8 & 28.8 & 15.4 & & & 3.8 & 1.9 & & & & 100.0 \\
\hline Ertapenem & 11.5 & 26.9 & 23.1 & 15.4 & 3.8 & 5.8 & 3.8 & 1.9 & 5.8 & 1.9 & & & 98.1 \\
\hline \multicolumn{14}{|l|}{ Klebsiella spp. (79) } \\
\hline Imipenem & & & & 3.8 & 36.7 & 50.6 & 3.8 & 3.8 & 1.3 & & & & 100.0 \\
\hline Meropenem & & 3.8 & 31.6 & 53.2 & 10.1 & & & & 1.3 & & & & 100.0 \\
\hline Ertapenem & & 16.5 & 21.5 & 20.3 & 30.4 & 10.1 & & & & & & 1.3 & 98.7 \\
\hline \multicolumn{14}{|l|}{ All isolates. (131) } \\
\hline Imipenem & & & & 3.8 & 35.1 & 48.9 & 7.6 & 2.3 & 2.3 & & & & 100.0 \\
\hline Meropenem & & 10.7 & 30.5 & 43.5 & 12.2 & & & 1.5 & 1.5 & & & & 100.0 \\
\hline Ertapenem & 4.6 & 20.6 & 22.1 & 18.3 & 19.8 & 8.4 & 1.5 & 0.8 & 2.3 & 0.8 & & 0.8 & 98.5 \\
\hline
\end{tabular}

${ }^{a}$ Values of $0 \%$ are left intentionally blank. No isolates exceeded an MIC of $16 \mu \mathrm{g} / \mathrm{ml}$.

${ }^{b}$ Susceptibility determined using 2005 CLSI breakpoints, as follows: $4 \mu \mathrm{g} / \mathrm{ml}$ for imipenem and meropenem; 2 $\mu \mathrm{g} / \mathrm{ml}$ for ertapenem.

listed in Table 1. The frequency distribution of MIC values for each antibiotic was used as a discrete probability density function for purposes of the simulations.

Pharmacokinetics. Pharmacokinetic data were obtained from previously published studies in healthy volunteers. ${ }^{[18-21]}$ For studies to be considered, they had to have: 1) been conducted using at least 10 healthy volunteers; 2) described the assay used to determine drug concentrations; 3) used clinically relevant dosing regimens; 4) performed an adequate pharmacokinetic analysis as determined by OPTAMA investigators and 5) presented means and standard deviations for total body clearance $\left(C L_{T}\right)$ and volume of distribution $\left(V_{d}\right)$. For studies reporting pharmacokinetic profiles as a two compartment model, the $V_{d \beta}$ was calculated from the terminal elimination rate constant for use in the one compartment model. Values for these parameters are listed in Table 2. $C L_{T}$ and $V_{d}$ were assumed to follow log-gaussian distrbutions during simulations.

Estimates of the fraction unbound $\left(f_{u}\right)$ for all drugs evaluated were derived from the package insert for each antibiotic and from the other studies previously described. The unbound fractions for these agents were treated as ranges and are also listed in Table 2. Since there are no data to suggest that these estimates are described by any particular distribution, it was assumed that the fraction unbound followed a uniform distribution whereby any value within the simulated range had an equal probability of occurring.
Table 2: Summary of pharmacokinetic parameters and variability for antimicrobials used in the Monte Carlo simulation.

\begin{tabular}{lccc}
\hline & \multicolumn{3}{c}{ Pharmacokinetic parameter (mean $\pm \mathrm{SD})$} \\
\cline { 2 - 4 } Antibiotic & $C L_{T}(\text { liters } / \mathrm{h})^{a}$ & $V_{d}$ (liters) $^{a}$ & $f_{u}(\%)^{b}$ \\
\hline Imipenem & $10.5 \pm 1.4$ & $17.2 \pm 3.8$ & $80-95$ \\
Meropenem & $14.4 \pm 1.8$ & $22.7 \pm 3.4$ & $85-98$ \\
Ertapenem & $1.2 \pm 0.19$ & $7.5 \pm 1.5$ & $5-15$ \\
\hline
\end{tabular}

${ }^{a}$ Results are expressed as means \pm standard deviations.

${ }^{b}$ Fraction unbound, presented as a range.

Monte Carlo Simulation. Three 5,000 patient Monte Carlo simulations using Crystal Ball 2000 (Decisioneering, Inc., Denver, CO, USA) were conducted in order to calculate estimates of $f \mathrm{~T}_{>\text {MIC }}$ for each antibiotic regimen/bacterial population combination. Each compound and dose was modeled against the E. coli isolates, against the Klebsiella spp. isolates, and against all ESBL isolates as a group. During each iteration, different values for $C L_{T}, V_{d}, f_{u}$ and $M I C$ were substituted into the appropriate equations based on the probability density function for each, thereby resulting in 5,000 different estimates of pharmacodynamic exposure for each antibiotic regimen against each bacterial population. The cumulative fraction of response (CFR) was calculated for each compound at an $f \mathrm{~T}_{>\mathrm{MIC}}$ of 20,30 , 40 , and $50 \%$. CFR is the expected population probability of target attainment for a specific drug 
and dose against a specific population of microorganisms. [22] It incorporates both the probability of target attainment (PTA) for the drug under consideration at any given MIC (a concept independent of the organism being treated) and the MIC distribution of the organism of interest. Since Crystal Ball enables the user to conduct the simulation without displaying the PTA, the investigators derived the PTA for each regimen separately in order to evaluate its influence on the results. For all carbapenems, a pharmacodynamic breakpoint of $40 \% f \mathrm{~T}_{>\mathrm{MIC}}$ was considered bactericidal.

Confidence intervals for proportions were calculated at $\alpha=.05$ using the Newcombe-Wilson method without correction for continuity. [23] Statistical significance was evaluated using the chisquared method for a difference between two proportions (SigmaStat 2.0.3, Chicago, IL).

\section{RESULTS}

The bactericidal PTA for each regimen is shown in Figure 1. Ertapenem exhibited a probability of target attainment $40 \% \mathrm{fT}_{>\mathrm{MIC}}$ of $77 \%$ at an MIC of $2 \mu \mathrm{g} / \mathrm{ml}-$ its CLSI susceptibility breakpoint. Probabilities of achieving $40 \% f \mathrm{~T}_{>\text {MIC }}$ of $96 \%$ and $90 \%$ were observed for imipenem and meropenem, respectively, at their CLSI breakpoint of $4 \mu \mathrm{g} / \mathrm{ml}$. Cumulative fraction of response for the antimicrobial regimens tested are shown in Table 3. Both imipenem and meropenem achieved the pharmacodynamic target of $40 \% f \mathrm{~T}_{>\mathrm{MIC}}$ in all trials simulated. Meropenem exhibited a CFR of $100 \%$ (95\% CI 99.9,100.0) against E. coli organisms alone, against Klebsiella organisms alone, and against all ESBLs modeled together. Imipenem was equally effective against $E$. coli species with a CFR of $100.0 \%$ (95\%CI 99.9,100.0); it further demonstrated a $100 \%$ probability of achieving $40 \% \mathrm{f}_{>\mathrm{MIC}}$ against Klebsiella species and also against all ESBLs together.

Ertapenem also displayed excellent efficacy against the modeled pathogens, although slightly lower than the other carbapenems, with a CFR of $96.9 \%$ (95\% CI 96.4,97.4) against $E$. coli alone, $98.7 \%$ (95\% CI 98.3,98.9) against Klebsiella spp. alone, and 98.1\% $(95 \%$ CI 97.7,98.5) against all isolates grouped together. The difference between ertapenem's CFR and the CFR of each of the other agents was statistically significant $(\mathrm{p}<0.001)$.

\section{DISCUSSION}

Numerous hospitals have added ertapenem to the formulary for the treatment of Escherichia coli and

Table 3: Cumulative fraction of response at varying $f \mathrm{~T}_{>\mathrm{MIC}}$ exposures for carbapenems tested against ESBL producing bacteria isolated at 13 hospitals in North America.

\begin{tabular}{|c|c|c|c|c|c|}
\hline \multirow{2}{*}{$\begin{array}{l}\text { Species (no.) or } \\
\text { antimicrobial agent }\end{array}$} & \multicolumn{4}{|c|}{ CFR at varying $f \mathrm{~T}_{>\mathrm{MIC}}$ exposures $^{a}$} & \multirow[t]{2}{*}{$95 \% \mathrm{CI}$ at $40 \% f \mathrm{~T}_{>\mathrm{MIC}}{ }^{b}$ : } \\
\hline & $20 \%$ & $30 \%$ & $40 \%$ & $50 \%$ & \\
\hline E. coli $(52)$ & & & & & \\
\hline Imipenem $1 \mathrm{~g}$ q8h & 100.0 & 100.0 & 100.0 & 99.8 & $(99.9,100.0)$ \\
\hline Meropenem $1 \mathrm{~g} \mathrm{q} 8 \mathrm{~h}$ & 100.0 & 100.0 & 100.0 & 99.9 & $(99.9,100.0)$ \\
\hline Ertapenem 1g q $24 \mathrm{~h}$ & 99.7 & 98.7 & 96.9 & 94.6 & $(96.4,97.4)$ \\
\hline \multicolumn{6}{|l|}{ Klebsiella spp. (78) } \\
\hline Imipenem 1g q8h & 100.0 & 100.0 & 100.0 & 99.9 & $(99.9,100.0)$ \\
\hline Meropenem 1g q8h & 100.0 & 100.0 & 100.0 & 99.9 & $(99.9,100.0)$ \\
\hline Ertapenem 1g q24h & 98.7 & 98.7 & 98.7 & 98.7 & $(98.3,98.9)$ \\
\hline \multicolumn{6}{|l|}{ All isolates (128) } \\
\hline Imipenem $1 \mathrm{~g} \mathrm{q} 8 \mathrm{~h}$ & 100.0 & 100.0 & 100.0 & 99.8 & $(99.9,100.0)$ \\
\hline Meropenem $1 \mathrm{~g} \mathrm{q} 8 \mathrm{~h}$ & 100.0 & 100.0 & 100.0 & 99.8 & $(99.9,100.0)$ \\
\hline Ertapenem $1 \mathrm{~g} \mathrm{q} 24 \mathrm{~h}$ & 99.1 & 98.8 & 98.1 & 97.0 & $(97.7,98.5)$ \\
\hline
\end{tabular}

${ }^{a} 40 \% f \mathrm{~T}_{>\mathrm{MIC}}$ is considered bactericidal for the carbapenems. The CFR at that level is displayed in bold type.

${ }^{b}$ Statistical significance was evaluated using the chi-squared test for a difference between two proportions. For all isolates at $40 \% f \mathrm{~T}_{>\mathrm{MIC}}$, the difference in the CFRs between ertapenem and imipenem and between ertapenem and meropenem was significant ( $\mathrm{p}<0.001$ for each pair). For Klebsiella spp., the difference in the CFRs between ertapenem and each other agent was significant $(\mathrm{p}<0.001$ for each pair). For $E$. coli, the difference in the CFRs between ertapenem and each other agent was significant ( $<<0.001$ for each pair). 
Klebsiella pneumoniae that produce ESBLs because it is more economical and more convenient, and because it may possibly avoid unnecessary selective pressure against Pseudomonas aeruginosa. However, recently published data have raised important questions about the comparative efficacy of ertapenem (versus the other carbapenems) against ESBLs. [6, 7] The presence of an ESBL increases the MIC to all of these antibiotics by at least two- to four-fold. While such increases are still within the range of susceptibility for imipenem and meropenem, in vitro data have observed that such an increase in MIC to ertapenem may fall outside of its susceptibility range.

In the absence of clinical data to document success or failure rates, the relative utility of the carbapenems can be evaluated based upon their ability to obtain targeted pharmacodynamic endpoints. A recent study by Moczygemba, et al. compared all three carbapenems against a small number (39 isolates) of ESBL producing bacteria collected from University Hospital in San Antonio, Texas. [8] Using pharmacodynamic modeling with an IV bolus equation, these investigators observed that imipenem and meropenem had a much greater likelihood of obtaining $40 \% f \mathrm{~T}_{>\mathrm{MIC}}(98 \%$ and $97 \%$, respectively) than ertapenem $(78 \%)$. That examination reported susceptibility of ESBLs to ertapenem of $87 \%$, versus $100 \%$ and $97 \%$ to imipenem and meropenem, respectively. However, the investigation did not distinguish results between pathogens, and clearly observed much higher rates of ertapenem resistance than found in other reports, including ours. [24]

The present study assessed the performance of carbapenems against ESBLs collected from a broad geographic area over a defined time period, with the aim of discriminating among the three compounds. Because resistance rates vary not only geographically, but also among different hospitals in the same city, the isolates were collected from as many sites as possible in an attempt to create a nationally representative sample. While the ability to cleave $\beta$-lactam rings is the ESBLs' primary mechanism of resistance to antimicrobials, other forms of resistance may be developing and may be present in some species, but not others. In order to illuminate that possibility, the study conducted three simulations of 5,000 trials each: one against E. coli alone (52 isolates), one against Klebsiella spp. alone (79 isolates), and one against the two groups of isolates combined (131 isolates total). Samples included were collected no earlier than 2002 to ensure a timely analysis.

Standard susceptibility testing is the most common method of determining the efficacy of a compound against pathogens of interest. Indeed, such testing is an important part of surveillance programs such as MYSTIC that attempt to document current antibacterial potency around the world. However, MIC data alone are inadequate to appropriately inform clinical judgment regarding empiric antibiotic selection, as it is necessary to account also for variability in both the pharmacokinetic and pharmacodynamic properties of each antimicrobial agent. [11] The PTA analysis displayed in Figure 1 reveals that ertapenem has only a $77 \%$ probability of attaining $40 \% f \mathrm{~T}_{>\text {MIC }}$ at its CLSI breakpoint of $2 \mu \mathrm{g} / \mathrm{ml}$, which emphasizes the problematic nature of standard susceptibility testing. The susceptibility rates observed among the 131 isolates in the study sample using CLSI breakpoints were $98.5 \%$ to ertapenem and $100.0 \%$ to both imipenem and meropenem; these rates, interpreted alone, are likely to overpredict microbiological success.

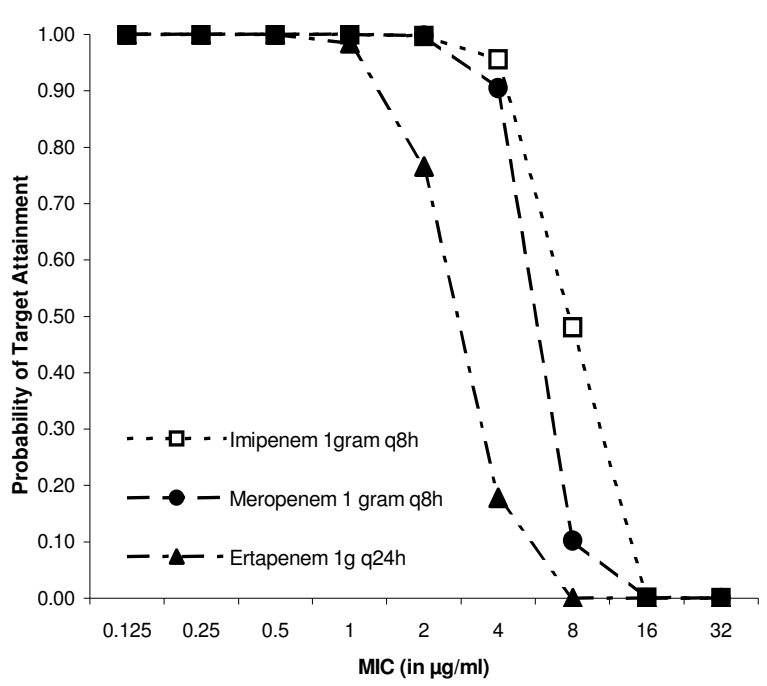

Fig 1: Probability of target attainment for the carbapenems at a target of $40 \% f \mathrm{~T}_{>\mathrm{MIC}}$

For the carbapenems, bactericidal activity against Gram negative organisms in animal models has been shown to correlate with free drug concentration remaining above the MIC for at least $40 \%$ of the dosing interval [25]; this target was chosen as an appropriate endpoint with which to analyze cumulative fraction of response. A range of $f \mathrm{~T}_{>\mathrm{MIC}}$ from $20 \%$ to $50 \%$ was evaluated to account for the possibility that the bactericidal breakpoint may be different for some subpopulations or may change over time. [26, 27] At $40 \% f \mathrm{~T}_{>\text {MIC }}$, both imipenem and meropenem displayed a CFR of $100 \%$ against ESBLs from both species modeled. These results suggest that imipenem and meropenem are equally likely to provide successful bactericidal coverage against ESBLs in the clinical setting. Ertapenem performed very competitively in the simulations, with a probability of achieving $40 \% f \mathrm{~T}_{>\mathrm{MIC}}$ above $96 \%$ against E. coli and above $98 \%$ for Klebsiella and for all ESBLs together. These results suggest that ertapenem, unlike in the Moczygemba study, has a high likelihood of providing bactericidal coverage against most ESBL producing organisms. Unlike the other compounds, however, ertapenem did not demonstrate a CFR of $100 \%$ for some trials at targets of $40 \% f \mathrm{~T}_{>\mathrm{MIC}}$ and below, and it did not spend 
any time above the MIC for a small number of trials (48 of 5000 trials $(0.96 \%)$ against Klebsiella spp, and 24 of 5000 trials $(0.48 \%)$ against E. coli and Klebsiella spp. combined). With regard to clinical significance, this suggests that a small number of patients nationwide may harbor ESBL organisms that cannot be treated with ertapenem.

In addition to prevalence of ESBLs, mechanisms of resistance must also be considered. The presence of ESBL production in and of itself is not predictive of efficacy. [28] A recently published study examining the resistance mechanisms of the carbapenems observed that ertapenem exhibited higher MICs for some Gramnegative isolates producing AmpC enzymes and ESBLs, as well as for isolates producing multiple $\beta$ lactamases. [24] The CFR observed for ertapenem against Klebsiella reflects the influence of one particularly resistant organism isolated from one of the MYSTIC sites. The exact mechanism of resistance of that isolate was not known, although initial examination revealed that it had an ESBL and a possible outer membrane porin alteration (R.N. Jones, personal communication). In this examination, only 2 of 131 isolates above ertapenem's breakpoint were enough to influence the results. The Moczygemba studyconducted with a considerably less susceptible group of organisms-showed comparatively worse ertapenem performance. While there are some differences in modeling techniques between the two studies (Moczygemba et al. used an IV bolus equation vs. our one-compartment, 30 minute infusion model; both used pharmacokinetic parameters from healthy volunteers), taken together they do suggest that ertapenem's ability to achieve pharmacodynamic targets is highly influenced by the MIC distribution in the organism population. Clinical and formulary decisions should account not only for elevated rates of ESBLs, but also for the possible presence (as detected by an institution's antibiogram) of organisms with mechanisms of resistance that may affect individual compounds in an antibiotic class differently. For some institutions, the potential for failed initial treatment may be worth considering as a part of the decision to designate any specific drug or class of drugs as first line therapy for ESBL infections.

In applying the results of this or any study to clinical practice, it is important to understand the limitations that occur when undertaking any exercise designed to model real-world conditions in a simulated environment. First, pharmacokinetic parameters were derived from healthy volunteer data. Variability of these parameters in the clinical setting is likely to be greater than that under study. Because reduced renal clearance in sicker patients could potentially increase $f \mathrm{~T}_{>\mathrm{MIC}}$, the authors believe that healthy volunteer data result in more conservative estimates of drug exposure. Further, such data is available for all the compounds under study, allowing a fair basis for comparison.
Second, the MIC distributions for the modeled pathogens at any given institution may be different than those examined here. The ideal simulation would use a Monte Carlo analysis that incorporated institutionspecific MIC data. The institutions included here are large teaching hospitals, and the results may be biased toward that setting. Third, the present study did not consider the "inoculum effect," which could raise the MICs to all agents tested [29]; MICs were tested using CLSI standards of $10^{5} \mathrm{CFU} / \mathrm{ml}$.

\section{CONCLUSION}

This analysis demonstrates that all three carbapenems-imipenem, meropenem and ertapenem-should perform capably against $E$. coli and Klebsiella species who produce ESBLs using any of their common dosing regimens. All three exhibited a high probability-often 100\%-of attaining bactericidal targets. However, ertapenem may not be the best choice for a first line agent against ESBLs in every institution. Organizations should examine their individual hospital antibiograms for high rates of ESBLs and/or MICs of greater than or equal to $2 \mu \mathrm{g} / \mathrm{ml}$ for those pathogens in order to determine if the reward of more convenient dosing and lesser expense is worth the risk of inappropriate initial therapy for a few patients harboring unusually resistant organisms.

\section{ACKNOWLEDGEMENTS}

We would like to acknowledge Ronald N. Jones and members of The Jones Group/JMI Laboratories (Iowa, USA) for performing susceptibility testing during the MYSTIC surveillance study and Philip J. Turner of AstraZeneca (Cheshire, U.K.) for his assistance with data management. We would also like to thank John Quinn, M.D. of Rush Presbyterian Medical Center (Chicago, IL) for providing data from isolates at his institution. The OPTAMA Program is made possible through a research grant from Astra-Zeneca Pharmaceuticals, Inc., Wilmington, DE.

\section{REFERENCES}

1. Bush, K., 2001. New beta-lactamases in gramnegative bacteria: diversity and impact on the selection of antimicrobial therapy. Clin Infect Dis, 32(7): 10859.

2. Knothe, H., et al., 1983. Transferable resistance to cefotaxime, cefoxitin, cefamandole and cefuroxime in clinical isolates of Klebsiella pneumoniae and Serratia marcescens. Infection, 11(6): 315-7.

3. Bradford, P.A., 2001. Extended-spectrum betalactamases in the 21st century: characterization, epidemiology, and detection of this important resistance threat. Clin Microbiol Rev, 14(4): 933-51, table of contents.

4. Paterson, D.L., et al., 2004. Antibiotic therapy for Klebsiella pneumoniae bacteremia: implications of production of extended-spectrum beta-lactamases. Clin Infect Dis, 39(1): 31-7. 
5. Gesser, R.M., et al., 2003. Efficacy of ertapenem in the treatment of serious infections caused by Enterobacteriaceae: analysis of pooled clinical trial data. J Antimicrob Chemother, 51(5): 1253-60.

6. Livermore, D.M., et al., 2001. Activity of ertapenem (MK-0826) versus Enterobacteriaceae with potent beta-lactamases. Antimicrob Agents Chemother, 45(10): 2831-7.

7. Livermore, D.M., et al., 2001. In vitro activities of ertapenem (MK-0826) against recent clinical bacteria collected in Europe and Australia. Antimicrob Agents Chemother, 45(6): 1860-7.

8. Moczygemba, L.R., C.R. Frei, and D.S. Burgess, 2004. Pharmacodynamic modeling of carbapenems and fluoroquinolones against bacteria that produce extended-spectrum beta-lactamases. Clin Ther, 26(11): 1800-7.

9. Craig,

W.A.,

1998.

Pharmacokinetic/pharmacodynamic parameters: rationale for antibacterial dosing of mice and men. Clin Infect Dis, 26(1): 1-10; quiz 11-2.

10. Bradley, J.S., M.N. Dudley, and G.L. Drusano, 2003. Predicting efficacy of antiinfectives with pharmacodynamics and Monte Carlo simulation. Pediatr Infect Dis J, 22(11): 982-92; quiz 993-5.

11. Mouton, J.W., 2002. Breakpoints: current practice and future perspectives. Int J Antimicrob Agents, 19(4): 323-31.

12. Kiffer, C.R., et al., 2004. Pharmacodynamic comparisons of antimicrobials against nosocomial isolates of escherichia coli, klebsiella pneumoniae, acinetobacter baumannii and pseudomonas aeruginosa from the MYSTIC surveillance program: the OPTAMA Program, South America 2002. Diagn Microbiol Infect Dis, 49(2): 109-16.

13. Kuti, J.L., C.H. Nightingale, and D.P. Nicolau, 2004. Optimizing pharmacodynamic target attainment using the MYSTIC antibiogram: data collected in North America in 2002. Antimicrob Agents Chemother, 48(7): 2464-70.

14. Masterton, R.G., et al., 2005. The OPTAMA programme: utilizing MYSTIC (2002) to predict critical pharmacodynamic target attainment against nosocomial pathogens in Europe. J Antimicrob Chemother, 55(1): 71-7.

15. Gibaldi, M., 1984. Introduction to Pharmacokinetics. In: Biopharmaceutics and Clinical Pharmacokinetics (ed. M. Gibaldi) pp 116. Lea \& Febiger, Philadelphia.

16. Rhomberg, P.R. and R.N. Jones, 2003. Antimicrobial spectrum of activity for meropenem and nine broad spectrum antimicrobials: report from the MYSTIC Program (2002) in North America. Diagn Microbiol Infect Dis, 47(1): 365-72.
17. Clinical and Laboratory Standards Institute. Methods for dilution antimicrobial susceptibilities tests for bacteria that grow aerobically-Fifteenth Informational Supplement. CLSI/NCCLS document M100-S15. Clinical and Laboratory Standards Institute, 940 West Valley Road, Suite 1400, Wayne, Pennsylvania 19807-1898 USA, 2005.

18. Pletz, M.W., et al., 2004. Ertapenem pharmacokinetics and impact on intestinal microflora, in comparison to those of ceftriaxone, after multiple dosing in male and female volunteers. Antimicrob Agents Chemother, 48(10): 3765-72.

19. Kuti, J.L., et al., 2005. Comparison of pharmacodynamic target attainment between healthy subjects and patients for ceftazidime and meropenem. Pharmacotherapy, 25(7): 935-41.

20. Mouton, J.W., N. Punt, and A.A. Vinks, 2005. A retrospective analysis using Monte Carlo simulation to evaluate recommended ceftazidime dosing regimens in healthy volunteers, patients with cystic fibrosis, and patients in the intensive care unit. Clin Ther, 27(6): 762-72.

21. Dreetz, M., et al., 1996. Serum bactericidal activities and comparative pharmacokinetics of meropenem and imipenem-cilastatin. Antimicrob Agents Chemother, 40(1): 105-9.

22. Mouton, J.W., et al., 2005. Standardization of pharmacokinetic/pharmacodynamic (PK/PD) terminology for anti-infective drugs: an update. $\mathrm{J}$ Antimicrob Chemother, 55(5): 601-7.

23. Newcombe, R.G., 1998. Two-sided confidence intervals for the single proportion: comparison of seven methods. Stat Med, 17(8): 857-72.

24. Jones, R.N., H.S. Sader, and T.R. Fritsche, 2005. Comparative activity of doripenem and three other carbapenems tested against Gram-negative bacilli with various beta-lactamase resistance mechanisms. Diagn Microbiol Infect Dis, 52(1): 71-4.

25. Mattoes, H.M., et al., 2004. Optimizing antimicrobial pharmacodynamics: dosage strategies for meropenem. Clin Ther, 26(8): 1187-98.

26. Drusano, G.L., 2003. Prevention of resistance: a goal for dose selection for antimicrobial agents. Clin Infect Dis, 36(Suppl 1): S42-50.

27. Turnidge, J.D., 1998. The pharmacodynamics of beta-lactams. Clin Infect Dis, 27(1): 10-22.

28. Ambrose, P.G., S.M. Bhavnani, and R.N. Jones, 2003. Pharmacokinetics-pharmacodynamics of cefepime and piperacillin-tazobactam against Escherichia coli and Klebsiella pneumoniae strains producing extended-spectrum beta-lactamases: report from the ARREST program. Antimicrob Agents Chemother, 47(5): 1643-6.

29. Thomson, K.S. and E.S. Moland, 2001. Cefepime, piperacillin-tazobactam, and the inoculum effect in tests with extended-spectrum beta-lactamaseproducing Enterobacteriaceae. Antimicrob Agents Chemother, 45(12): 3548-54. 